A Hard Constraint Algorithm to Model Particle Interactions in DNA-laden Flows

D. Trebotich, G. H. Miller, M. D. Bybee

August 2, 2006

Nanoscale and Microscale Thermophysical Engineering 
This document was prepared as an account of work sponsored by an agency of the United States Government. Neither the United States Government nor the University of California nor any of their employees, makes any warranty, express or implied, or assumes any legal liability or responsibility for the accuracy, completeness, or usefulness of any information, apparatus, product, or process disclosed, or represents that its use would not infringe privately owned rights. Reference herein to any specific commercial product, process, or service by trade name, trademark, manufacturer, or otherwise, does not necessarily constitute or imply its endorsement, recommendation, or favoring by the United States Government or the University of California. The views and opinions of authors expressed herein do not necessarily state or reflect those of the United States Government or the University of California, and shall not be used for advertising or product endorsement purposes. 


\title{
A Hard Constraint Algorithm to Model Particle Interactions in DNA-laden Flows
}

\author{
D. Trebotich* \\ Center for Applied Scientific Computing, Lawrence Livermore National \\ Laboratory, P.O. Box 808, L-560, Livermore, CA 94551, USA \\ G. H. Miller \\ Department of Applied Science, University of California, One Shields Avenue, \\ Davis, CA 95616, USA \\ M. D. Bybee \\ Department of Chemical and Biomolecular Engineering, University of Illinois at \\ Urbana-Champaign, Urbana, IL 61801, USA
}

\begin{abstract}
We present a new method for particle interactions in polymer models of DNA. The DNA is represented by a bead-rod polymer model and is fully-coupled to the fluid. The main objective in this work is to implement short-range forces to properly model polymer-polymer and polymer-surface interactions, specifically, rod-rod and rod-surface uncrossing. Our new method is based on a rigid constraint algorithm whereby rods elastically bounce off one another to prevent crossing, similar to our previous algorithm used to model polymer-surface interactions. We compare this model to a classical (smooth) potential which acts as a repulsive force between rods, and rods and surfaces.
\end{abstract}

\section{Introduction}

Microfluidic devices are increasingly important in biodefense and biomedical applications including pathogen detection, continuous monitoring, and drug

\footnotetext{
* Corresponding author.

Email addresses: trebotich1@llnl.gov (D. Trebotich), grgmiller@ucdavis.edu (G. H. Miller), bybee@uiuc.edu (M. D. Bybee).
} 
delivery. Numerical algorithms that can model flows of complex biological fluids within these devices are needed for further development and optimization. For example, a technique for "sticking" is necessary to model the capture of DNA in an extraction chamber from whence a polymerase chain reaction can begin. This phenomenon requires modeling of short-range interactions between large molecules and a (smooth) structural surface. In addition, biological fluids are usually polymeric, a solvent consisting of multiple, large particles which undergo self-interactions as well as interactions with other particles. The polymer model and these short-range interactions must be physically appropriate for there to be fidelity in the simulation of transport. Furthermore, the problem is complicated due to the time scale of the short-range interactions being much smaller than that of the bulk fluid, necessitating multiscale schemes.

In previous work we developed a model that coupled bead-rod polymers to an incompressible viscous solvent [9]. We took care to conservatively couple the polymer and solvent forces - both viscous and stochastic - in order that the fluid "feel" the effect of the polymer. It is in this sense of obeying Newton's third law of motion that we consider the dynamics to be "tightly coupled". (The purpose of the full-coupling is to be able to simulate the effects of a large number of polymers and to compare this hybrid approach with a viscoelastic continuum model as in [7].) Additionally, the polymer nodes may experience elastic collisions with domain boundaries. With this numerical algorithm, we have been able to simulate polymer-boundary interactions which occur in DNA size-separation and extraction devices as well as obtain preliminary results for more complicated 2D and 3D device geometries [8]. The model also captures many essential features observed in DNA visualization experiments [3]. In particular, the molecule tends to extend in regions of large shear flow, and contract in its absence.

However, to model the (probable) fate of individual molecules in microfluidic systems or biological flows, it is desirable to incorporate more physicallyrealistic behavior. For polymer models of DNA, of immediate concern is the non-crossing constraint: a polymer section cannot pass through another polymer section. In our previous freely-jointed bead-rod model, as with many other current implementations (e.g., [6]), crossing of rod sections is allowed. We treated polymer-surface interactions as purely elastic collisions, and we do not treat polymer-polymer interactions. In particular, there is no rod-rod non-crossing constraint. The resulting behavior has a strong theoretical foundation (e.g., [4]) and is therefore important for algorithm validation, but does not respect the correct non-crossing physical behavior of real molecules.

Furthermore, macromolecules like DNA are charged, and chemically active. They interact through screened Coulombic interactions and migrate in response to imposed electric fields. In addition, microfluidic separators have been designed based on the increase of residence time with molecule length in packed 
bed and pillared array geometries, or through chemically-mediated residence time enhancement achieved by binding selective proteins to channel surfaces. These physical effects are characterized by intra-polymer, inter-polymer, and polymer-surface interaction potentials which may be long-ranged.

In this paper we will explore the rod-crossing problem using rigid constraints. We will also implement a soft penalty method where Coulombic interactions using a short-range Debye-Hückel potential are incorporated into a classic repulsive potential. A preliminary version of this work can be found in [1].

\section{Algorithmic Approach}

Two methods for preventing rod-rod crossings in bead-rod polymer models are presented here. The first is a new hard constraint mechanism for elastically bouncing rigid rods off of each other. This approach to rod-rod uncrossing is currently used to prevent rod-surface crossing in microfluidic post array channels [9]. The second is a repulsive potential as in [2] to model short-range rod-rod and rod-surface interactions for comparison to the hard constraint system.

\subsection{Rod-rod Uncrossability Constraint}

The rod-rod uncrossability constraint works by detecting rod-rod collisions and treating them as elastic collisions between infinitely thin rods. It is similar to the bead-surface uncrossability constraint already implemented in [9]. For simplicity in exposition the algorithm in 2D is outlined below as an extension to the existing algorithm in [9]. Steps 1, 2, and 4 are described in more detail in [9]. The ideas for steps 3a-3d have been taken from [5]. In that work bonds are considered as elastic bands between bonded particles. When any two of these elastic bands make contact, an entanglement point is created which prevents them from crossing. We call this a hard constraint algorithm as it is an end member of a classical smooth potential that requires no parameter tuning.

The polymer is represented by a series of beads connected by rigid rods. The bead positions and velocities are given by $\boldsymbol{x}$ and $\boldsymbol{v}$ respectively. Rod $i$ is defined as the line segment from bead $i$ to bead $i+1$, or from $\boldsymbol{x}_{i}$ to $\boldsymbol{x}_{i+1}$.

For each time step, beginning with $\boldsymbol{x}^{n}$ and $\boldsymbol{v}^{n}$ :

1. Calculate the unconstrained motion to obtain $\boldsymbol{x}^{*}$ and $\boldsymbol{v}^{*}$.

2. Calculate the motion subject to the rod length constraint to obtain $\boldsymbol{x}^{\dagger}$ and $\boldsymbol{v}^{\dagger}$. 
3. Calculate the motion subject to the rod-rod uncrossability constraint to obtain $\boldsymbol{x}^{\ddagger}$ and $\boldsymbol{v}^{\ddagger}$. The details of this step are as follows:

Calculate $\boldsymbol{v}^{\Delta t}$, the bead velocities over the current time step

$$
\boldsymbol{v}^{\Delta t}=\left(\boldsymbol{x}^{\dagger}-\boldsymbol{x}^{n}\right) / \Delta t
$$

so that the time-linear trajectory of each bead over the current time step is

$$
\boldsymbol{x}=\boldsymbol{x}^{n}+\boldsymbol{v}^{\Delta t} t \quad t \in(0, \Delta t)
$$

Repeatedly loop through all rod pairs until no more collisions are detected. For each pair of rods $i$ and $j$ :

3a. Calculate the triple product $V_{i j}$ at times 0 and $\Delta t$ where

$$
V_{i j}=\left(\boldsymbol{x}_{i}-\boldsymbol{x}_{j}\right) \cdot\left(\left(\boldsymbol{x}_{i+1}-\boldsymbol{x}_{i}\right) \times\left(\boldsymbol{x}_{j+1}-\boldsymbol{x}_{j}\right)\right)
$$

The value of $V_{i j}$ will be zero if the infinite lines containing the rods intersect or are parallel. Therefore, if the value of $V_{i j}$ changes sign over the time step, a possible rod-rod crossing has occurred. Otherwise, proceed to step $3 \mathrm{k}$.

3b. Calculate $\tau$, the time of crossing. Substituting the time-linear trajectories of (1) into (2) gives a third-order polynomial in $t$ for $V_{i j}$. The smallest root of this polynomial in the range $(0, \Delta t)$ will be $\tau$.

To simplify the calculation, let

$$
\begin{aligned}
\boldsymbol{\alpha} & =\boldsymbol{x}_{i}-\boldsymbol{x}_{j}=\boldsymbol{\alpha}_{0}+\boldsymbol{\alpha}_{1} t \\
\boldsymbol{\beta} & =\boldsymbol{x}_{i+1}-\boldsymbol{x}_{i}=\boldsymbol{\beta}_{0}+\boldsymbol{\beta}_{1} t \\
\boldsymbol{\gamma} & =\boldsymbol{x}_{j+1}-\boldsymbol{x}_{j}=\gamma_{0}+\boldsymbol{\gamma}_{1} t
\end{aligned}
$$

where

$$
\begin{aligned}
\boldsymbol{\alpha}_{0} & =\boldsymbol{x}_{i}^{n}-\boldsymbol{x}_{j}^{n} \\
\boldsymbol{\alpha}_{1} & =\boldsymbol{v}_{i}^{\Delta t}-\boldsymbol{v}_{j}^{\Delta t} \\
\boldsymbol{\beta}_{0} & =\boldsymbol{x}_{i+1}^{n}-\boldsymbol{x}_{i}^{n} \\
\boldsymbol{\beta}_{1} & =\boldsymbol{v}_{i+1}^{\Delta t}-\boldsymbol{v}_{i}^{\Delta t} \\
\gamma_{0} & =\boldsymbol{x}_{j+1}^{n}-\boldsymbol{x}_{j}^{n} \\
\gamma_{1} & =\boldsymbol{v}_{j+1}^{\Delta t}-\boldsymbol{v}_{j}^{\Delta t}
\end{aligned}
$$

The coefficients of the polynomial $V_{i j}=a_{3} t^{3}+a_{2} t^{2}+a_{1} t+a_{0}$ are then given by

$$
\begin{aligned}
& a_{0}=\boldsymbol{\alpha}_{\mathbf{0}} \cdot\left(\boldsymbol{\beta}_{\mathbf{0}} \times \gamma_{\mathbf{0}}\right) \\
& a_{1}=\boldsymbol{\alpha}_{\mathbf{0}} \cdot\left(\boldsymbol{\beta}_{\mathbf{1}} \times \gamma_{\mathbf{0}}\right)+\boldsymbol{\alpha}_{\mathbf{1}} \cdot\left(\boldsymbol{\beta}_{\mathbf{0}} \times \gamma_{\mathbf{0}}\right)+\boldsymbol{\alpha}_{\mathbf{0}} \cdot\left(\boldsymbol{\beta}_{\mathbf{0}} \times \gamma_{\mathbf{1}}\right) \\
& a_{2}=\boldsymbol{\alpha}_{\mathbf{1}} \cdot\left(\boldsymbol{\beta}_{\mathbf{1}} \times \gamma_{\mathbf{0}}\right)+\boldsymbol{\alpha}_{\mathbf{0}} \cdot\left(\boldsymbol{\beta}_{\mathbf{1}} \times \gamma_{\mathbf{1}}\right)+\boldsymbol{\alpha}_{\mathbf{1}} \cdot\left(\boldsymbol{\beta}_{\mathbf{0}} \times \gamma_{\mathbf{1}}\right) \\
& a_{3}=\boldsymbol{\alpha}_{\mathbf{1}} \cdot\left(\boldsymbol{\beta}_{\mathbf{1}} \times \gamma_{\mathbf{1}}\right)
\end{aligned}
$$


3c. Calculate $\boldsymbol{x}^{\tau}$, the bead positions at time $\tau$.

$$
\boldsymbol{x}^{\tau}=\boldsymbol{x}^{n}+\boldsymbol{v}^{\Delta t} \tau
$$

3d. Calculate $\chi$, the point of intersection at time $\tau$, by solving the following set of equations:

$$
\chi=\boldsymbol{x}_{i}^{\tau}+\lambda_{i}\left(\boldsymbol{x}_{i+1}^{\tau}-\boldsymbol{x}_{i}^{\tau}\right)=\boldsymbol{x}_{j}^{\tau}+\lambda_{j}\left(\boldsymbol{x}_{j+1}^{\tau}-\boldsymbol{x}_{j}^{\tau}\right)
$$

where $\lambda_{i}$ and $\lambda_{j}$ define the point of intersection between the lines containing rods $i$ and $j$ respectively. If $\left(0 \leq \lambda_{i} \leq 1\right)$ and $\left(0 \leq \lambda_{j} \leq 1\right)$ then the point of intersection lies on both rods and a rod-rod crossing has occurred. Otherwise, proceed to step 3k.

3e. Calculate $\boldsymbol{n}$, the unit vector normal to the plane formed by the two rods at time $\tau$

$$
\boldsymbol{n}=\frac{\left(\boldsymbol{x}_{i+1}^{\tau}-\boldsymbol{x}_{i}^{\tau}\right) \times\left(\boldsymbol{x}_{j+1}^{\tau}-\boldsymbol{x}_{j}^{\tau}\right)}{\left|\left(\boldsymbol{x}_{i+1}^{\tau}-\boldsymbol{x}_{i}^{\tau}\right) \times\left(\boldsymbol{x}_{j+1}^{\tau}-\boldsymbol{x}_{j}^{\tau}\right)\right|}
$$

3f. Calculate $\boldsymbol{v}_{\text {rel }}^{\Delta t}$, the relative velocity of the intersection point over the current time step

$$
\boldsymbol{v}_{\text {rel }}^{\Delta t}=\left(\boldsymbol{v}_{j}^{\Delta t}-\boldsymbol{v}_{i}^{\Delta t}\right)+\lambda_{j}\left(\boldsymbol{v}_{j+1}^{\Delta t}-\boldsymbol{v}_{j}^{\Delta t}\right)-\lambda_{i}\left(\boldsymbol{v}_{i+1}^{\Delta t}-\boldsymbol{v}_{i}^{\Delta t}\right)
$$

3g. Calculate $\boldsymbol{v}^{\mathrm{col}}$, bead velocities after collision

$$
\begin{aligned}
\boldsymbol{v}_{i}^{\mathrm{col}} & =\boldsymbol{v}_{i}^{\Delta t}+2\left(1-\lambda_{i}\right)\left(\boldsymbol{n} \cdot \boldsymbol{v}_{\mathrm{rel}}^{\Delta t}\right) \boldsymbol{n} \\
\boldsymbol{v}_{i+1}^{\mathrm{col}} & =\boldsymbol{v}_{i+1}^{\Delta t}+2 \lambda_{i}\left(\boldsymbol{n} \cdot \boldsymbol{v}_{\mathrm{rel}}^{\Delta t}\right) \boldsymbol{n} \\
\boldsymbol{v}_{j}^{\mathrm{col}} & =\boldsymbol{v}_{j}^{\Delta t}-2\left(1-\lambda_{j}\right)\left(\boldsymbol{n} \cdot \boldsymbol{v}_{\mathrm{rel}}^{\Delta t}\right) \boldsymbol{n} \\
\boldsymbol{v}_{j+1}^{\mathrm{col}} & =\boldsymbol{v}_{j+1}^{\Delta t}-2 \lambda_{j}\left(\boldsymbol{n} \cdot \boldsymbol{v}_{\mathrm{rel}}^{\Delta t}\right) \boldsymbol{n}
\end{aligned}
$$

3h. Update positions for beads $i, i+1, j$, and $j+1$

$$
\boldsymbol{x}^{\ddagger}=\boldsymbol{x}^{\tau}+(\Delta t-\tau) \boldsymbol{v}^{\mathrm{col}}
$$

3i. Calculate $\boldsymbol{v}_{\text {rel }}^{\dagger}$, the relative velocity of the intersection point using velocities at the end of time step

$$
\boldsymbol{v}_{\mathrm{rel}}^{\dagger}=\left(\boldsymbol{v}_{j}^{\dagger}-\boldsymbol{v}_{i}^{\dagger}\right)+\lambda_{j}\left(\boldsymbol{v}_{j+1}^{\dagger}-\boldsymbol{v}_{j}^{\dagger}\right)-\lambda_{i}\left(\boldsymbol{v}_{i+1}^{\dagger}-\boldsymbol{v}_{i}^{\dagger}\right)
$$

3j. Update bead velocities at the end of the time step

$$
\begin{aligned}
\boldsymbol{v}_{i}^{\ddagger} & =\boldsymbol{v}_{i}^{\dagger}+2\left(1-\lambda_{i}\right)\left(\boldsymbol{n} \cdot \boldsymbol{v}_{\mathrm{rel}}^{\dagger}\right) \boldsymbol{n} \\
\boldsymbol{v}_{i+1}^{\ddagger} & =\boldsymbol{v}_{i+1}^{\dagger}+2 \lambda_{i}\left(\boldsymbol{n} \cdot \boldsymbol{v}_{\mathrm{rel}}^{\dagger}\right) \boldsymbol{n} \\
\boldsymbol{v}_{j}^{\ddagger} & =\boldsymbol{v}_{j}^{\dagger}-2\left(1-\lambda_{j}\right)\left(\boldsymbol{n} \cdot \boldsymbol{v}_{\mathrm{rel}}^{\dagger}\right) \boldsymbol{n} \\
\boldsymbol{v}_{j+1}^{\ddagger} & =\boldsymbol{v}_{j+1}^{\dagger}-2 \lambda_{j}\left(\boldsymbol{n} \cdot \boldsymbol{v}_{\mathrm{rel}}^{\dagger}\right) \boldsymbol{n}
\end{aligned}
$$


3k. If no collision occurs, then for beads $i, i+1, j$, and $j+1$

$$
\begin{aligned}
& \boldsymbol{v}^{\ddagger}=\boldsymbol{v}^{\dagger} \\
& \boldsymbol{x}^{\ddagger}=\boldsymbol{x}^{\dagger}
\end{aligned}
$$

4. Calculate the motion subject to the bead-surface uncrossability constraint to obtain $\boldsymbol{x}^{n+1}$ and $\boldsymbol{v}^{n+1}$.

This 2D algorithm can be extended to 3D by considering bead-rod collisions where the bead is treated as a rod perpendicular to the $2 \mathrm{D}$ plane with its position on the $2 \mathrm{D}$ plane.

\subsection{Repulsive Potential}

The algorithm for a repulsive potential interaction between rods is taken from the work of Kumar and Larson [2]. The shortest vector between two rods is calculated, and a repulsive force is applied to both rods along that vector. While Kumar and Larson explored the use of an exponential potential and a Lennard-Jones potential, this work uses a short-ranged Debye-Hückel potential

$$
U^{\mathrm{DH}}=A \frac{e^{-\kappa r}}{r} .
$$

For polymer-surface interactions, the same algorithm was applied using the shortest vector between a rod and the level set boundary defining a surface. It should be noted that while the mathematically correct introduction of these forces is directly into the Langevin equation, we initially introduced these forces after the rod length constraint to avoid complexities in the Ito-Taylor expansion of $[9]$.

\section{Results and Discussion}

We simulated a variety of polymer lengths and combinations of rigid constraint and soft potential interactions. The polymer-surface interactions are based on the level set boundary; the flow geometry is based on the embedded boundary. For low grid resolution the difference between the embedded boundary and the level set boundary representations of a physical surface is visually obvious. For higher resolution the difference between the embedded boundary and the level set boundary is not visually obvious. Considering polymer-surface interactions, the repulsive potential is more stable, but the rigid constraint allows the polymer to get closer to the pillar resulting in slower motion due to lower fluid velocities near the boundary. In general, as the number of beads in the 

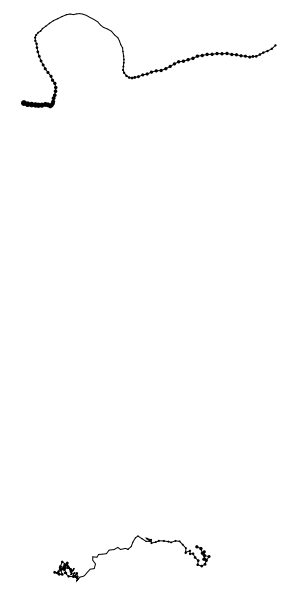
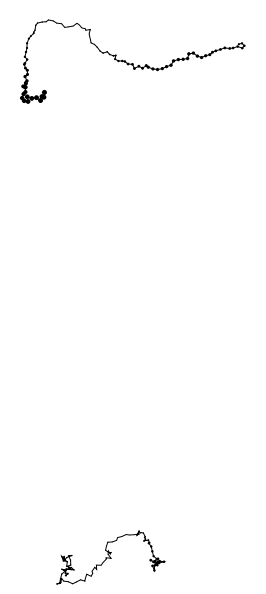

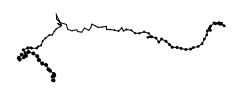

a

Fig. 1. Time sequence of a bead-rod polymer with varying particle masses in 3D. Polymer is initially stretched out but moves to a tangled state caused by transport of heavier particles up front in background flow.

polymer increased, the particle time step control had to be decreased in order to maintain convergence in the constant rod length constraint routine. This may be due to the beads being in a greater range of fluid velocities resulting in more stretching along the rods. For 100-bead polymers, a value of 1.0 was adequate; for 200-bead polymers, 0.1 .

In order to demonstrate the hard constraint algorithm in 3D we present results for the particle algorithm alone, decoupled from the time-dependent fluid algorithm. Figure 1 is a time sequence of a bead-rod polymer in 3D with varying particle (bead) masses being transported by a uniform background flow. Initially the polymer is in a relaxed, stretched out state. The heavier particles at the head cause the non-trivial behavior as the polymer begins to tangle in time.

Figure 2 is a time sequence of single polymer transport in a 2D microscale flow with a cylindrical pillar obstruction that demonstrates intra-polymer and polymer-structure interactions using the smooth potential. Time-dependent fluid coupling is included. In the first frame the polymer is randomly placed to be nearly entangled before flowing into the cylinder. Frame 2 shows a shortrange interaction of the polymer with the cylinder surface while also undergoing inter-polymer interactions. The polymer accelerates around the pillar in 

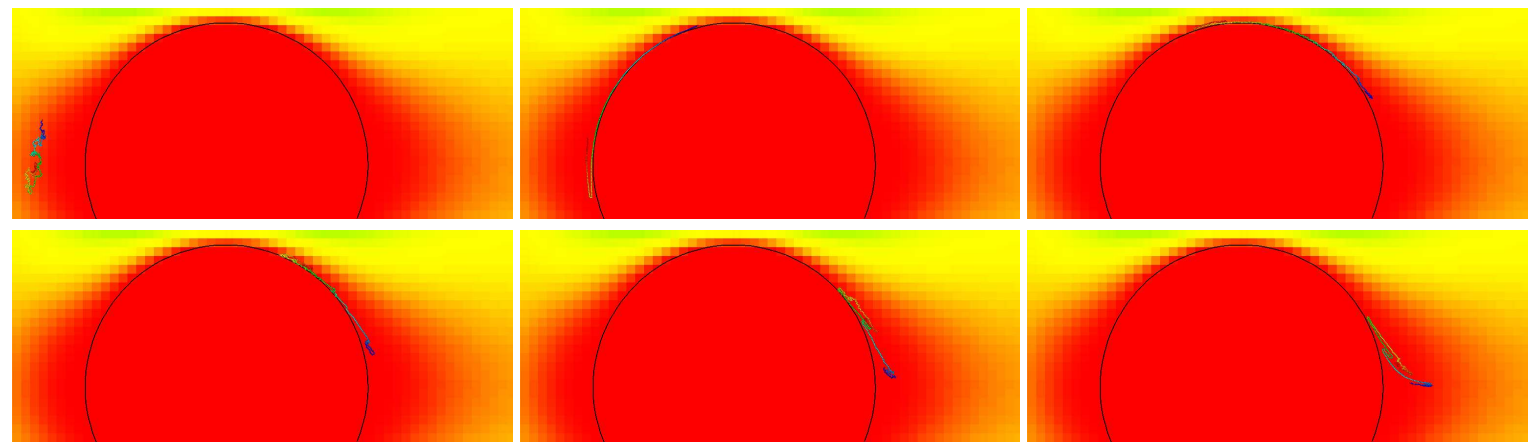

Fig. 2. Time sequence of 200-bead polymer flowing past a cylinder in 2D demonstrating intra-polymer and polymer-structure interactions using smooth potential. (a) Nearly entangled polymer. (b) Short-range polymer-surface interaction. (c) Acceleration around pillar due to Brownian perturbation and hydrodynamic drag. (d) More acceleration of the tail and slowing of the head in stagnation region in wake of pillar. (e) Accelerated tail catching up with stagnated head. (f) Re-entanglement in wake with multiple intra-polymer interactions.

the third and fourth frames due to Brownian perturbation keeping it off the surface and nudging it out into the boundary layer where it is pulled by hydrodynamic drag. As the polymer approaches the near-stagnation wake region behind the pillar the head of the polymer begins to slow down allowing the tail to catch up in the fifth and then sixth frames. Re-entanglement occurs in the wake where numerous intra-polymer interactions occur.

\section{Conclusion}

We have demonstrated a new method based on a rigid constraint system to prevent rod-crossing in bead-rod polymer models in 3D. The rigid constraint algorithm is the simplest model that captures the essential properties of roduncrossing. We use the same elastic collision model for rod-uncrossing as in polymer-surface interactions. The model has a physical basis in early molecular dynamics simulations where hard spheres represent condensed matter. On the other hand, the soft potential model involves arbitrary parameter tuning to determine the structure of the potential at from short to long range. The short range structure of the soft potential is also more restrictive on the particle time step than the hard constraint. The hard constraint is indeed an end member of the soft potential, eliminating the choice of parameters.

The new hard constraint algorithm does compare well qualitatively with the classical repulsive potential. It is difficult to see the differences during entanglement without establishing statistics from many simulations, which we did not perform here. Near surfaces, however, it is clear that the hard constraint allows the polymer to travel more closely to the surface where the fluid ve- 
locity is nearly zero. The smooth potential is currently computationally less expensive than the rigid constraint. Potentials need only be evaluated once per rod pair, whereas the rigid constraint must loop over and over until all collisions have been treated. Furthermore, the rod-rod uncrossability constraint loops through all rod-rod pairs treating collisions as they are found. This does not always lead to correct results. The algorithm still needs to be modified so that it searches all rod-rod pairs for the first occurring collision, treats it, then searches all rod-rod pairs for the next occurring collision, treats it, etc., being careful to maintain the proper $\boldsymbol{v}^{\Delta t}$ and $\boldsymbol{v}^{\text {col }}$. Combining the rod-rod and rod-surface uncrossability constraints into the same algorithm would be a more correct approach. There may also be exceptional cases not accounted for by the rod-rod uncrossability constraint (e.g., parallel rods). We introduced the forces resulting from potential interactions after the rod length constraint as a first attempt to avoid complexities in the Ito-Taylor expansion as in [9]. These forces should be introduced directly into the Langevin equation.

With the addition of these new force interactions, as expected, new highfrequency modes are introduced which limit stability and accuracy, necessitating adaptive time stepping strategies. Already we have seen this as a side-effect of the Rouse bead-spring polymer model. To address that problem we have considered decoupling the polymer and the fluid solvent time steps; this allows the relatively inexpensive polymer model to march forward with small time steps, resolving the high-frequency modes, while the very expensive fluid calculation marches forward with the greatest stable time step. Inclusion of long-range Coulombic interactions may increase the cost of polymer dynamics sufficiently that decoupling the time step is not a desirable strategy. Or, perhaps a partitioning of the Coulombic terms, as in the Ewald method, into short-range parts (resolved at the particle time scale) and long-range parts (resolved at the fluid time scale) can strike a good performance-accuracy trade-off.

\section{Acknowledgment}

This work was performed under the auspices of the U.S. Department of Energy by the University of California, Lawrence Livermore National Laboratory under contract No. W-7405-Eng-48. The work of G. H. Miller at the University of California, Davis was supported by LLNL IUT subcontract number B550201, and partially supported by DOE MICS contract number DE-FG02-03ER25579. The work of M. D. Bybee at the Lawrence Livermore National Laboratory was supported by the Department of Energy Computational Science Graduate Fellowship Program under grant number DE-FG0297ER25308. 


\section{References}

[1] M. D. Bybee, G. H. Miller, and D. Trebotich. Particle interactions in DNA flows. Technical Report UCRL-TR-217843, LLNL, December 2005.

[2] S. Kumar and R. G. Larson. Brownian dynamics simulations of flexible polymers with spring-spring repulsions. Journal of Chemical Physics, 114(15):6937-6941, 2001.

[3] E. C. Lee and S. J. Muller. Flow light scattering studies of polymer coil conformation in solutions in extensional flow. Macromolecules, 32:3295-3305, 1999.

[4] M. Mazars. Freely jointed chains in external potentials: analytical computations. J. Phys. A: Math. Gen., 32:1841-1861, 1999.

[5] J. T. Padding and W. J. Briels. Uncrossability constraints in mesoscopic polymer melt simulations: Non-Rouse behavior of $\mathrm{C}_{120} \mathrm{H}_{242}$. Journal of Chemical Physics, 115(6):2846-2859, 2001.

[6] M. Somasi, B. Khomami, N. J. Woo, J. S. Hur, and E. S. G. Shaqfeh. Brownian dynamics simulations of bead-rod and bead-spring chains: numerical algorithms and coarse-graining issues. Journal of Non-Newtonian Fluid Mechanics, 108:227$255,2002$.

[7] D. Trebotich, P. Colella, and G. H. Miller. A stable and convergent scheme for viscoelastic flow in contraction channels. J. Comp. Phys., 205:315-342, 2005.

[8] D. Trebotich and G. H. Miller. Modeling and simulation of DNA flow in a microfluidic-based pathogen detection system. In Proceedings of the 3rd Annual IEEE EMBS Special Topic Conference on Microtechnologies in Medicine and Biology, pages 353-355, 2005.

[9] D. Trebotich, G. H. Miller, P. Colella, D. T. Graves, D. F. Martin, and P. O. Schwartz. A tightly couple particle-fluid model for DNA-laden flows in complex microscale geometries. Computational Fluid and Solid Mechanics, pages 10181022, 2005. 